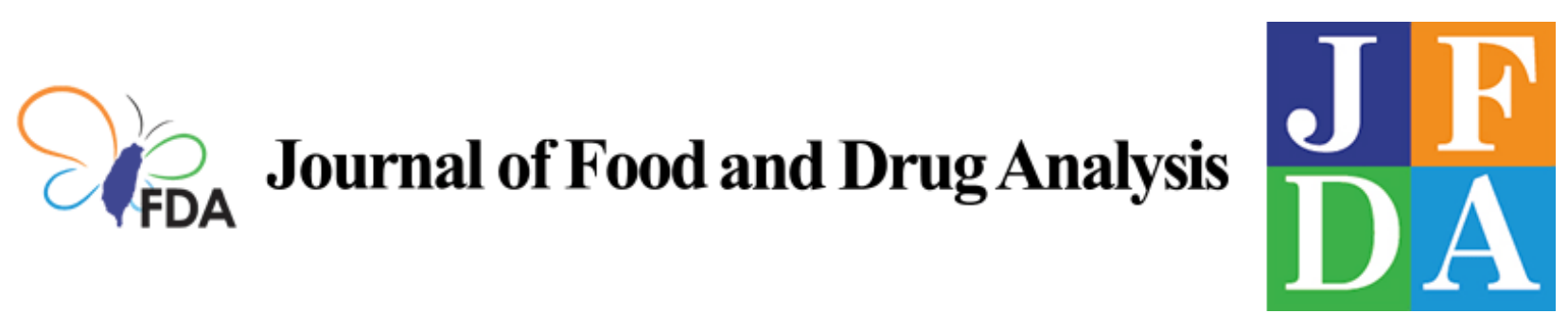

Volume 29 | Issue 2

Article 4

2021

\title{
Apigenin ameliorates hepatic lipid accumulation by activating the autophagy-mitochondria pathway
}

Follow this and additional works at: https://www.jfda-online.com/journal

Part of the Food Science Commons, and the Medicine and Health Sciences Commons (c) (i) (9)

This work is licensed under a Creative Commons Attribution-Noncommercial-No Derivative Works 4.0 License.

\section{Recommended Citation}

Hsu, Man Chen; Guo, Bei Chia; Hu, Po-An; Chen, Chia Hui; and Lee, Tzong-Shyuan (2021) "Apigenin ameliorates hepatic lipid accumulation by activating the autophagy-mitochondria pathway," Journal of Food and Drug Analysis: Vol. 29 : Iss. 2 , Article 4.

Available at: https://doi.org/10.38212/2224-6614.3269

This Original Article is brought to you for free and open access by Journal of Food and Drug Analysis. It has been accepted for inclusion in Journal of Food and Drug Analysis by an authorized editor of Journal of Food and Drug Analysis. 


\section{Apigenin ameliorates hepatic lipid accumulation by activating the autophagy- mitochondria pathway}

\section{Cover Page Footnote}

Acknowledgments This study was supported by grants from the Ministry of Science and Technology, Taiwan (106-2320-B-002-057-MY3, 106-2320-B-002-056, 106-2811-B-002-146; 108-2811-B-002-542 and 108-2320-B-002 -032-MY3). This article was subsidized for English editing by National Taiwan University under the Excellence Improvement Program for Doctoral Student (grant number 108-2926-I-002-002-MY4), sponsored by Ministry of Science and Technology, Taiwan. We thank the staff of the imaging core at the First Core Labs, National Taiwan University College of Medicine, for technical assistance. 


\title{
Apigenin ameliorates hepatic lipid accumulation by activating the autophagy-mitochondria pathway
}

\author{
Man-Chen Hsu, Bei-Chia Guo, Chia-Hui Chen, Po-An Hu, Tzong-Shyuan Lee*
}

Graduate Institute and Department of Physiology, College of Medicine, National Taiwan University, Taipei, Taiwan

\begin{abstract}
Apigenin, a flavonoid isolated from plants, provides protection against non-alcoholic fatty liver disease. However, the mechanism by which apigenin decreases lipid accumulation in the liver is unclear. In this study, we investigated the molecular mechanism underlying the beneficial effect of apigenin on the hepatic deregulation of lipid metabolism. Oleic acid (OA)-induced lipid accumulation in human hepatoma cells (Huh7 cells) was used as an in vitro model. Western blot analysis was used for evaluating protein expression. Oil red $\mathrm{O}$ staining, Nile red staining, and conventional assay kits were used to assess the level of lipids. Immunocytochemistry was performed to observe mitochondrial morphology. Seahorse XF analyzer was used to measure mitochondrial bioenergetics. Treatment with OA induced lipid accumulation in Huh7 cells, which was attenuated by apigenin. Mechanistically, treatment with apigenin increased the expression of autophagy-related proteins including Beclin1, autophagy related gene 5 (ATG5), ATG7, and LC3II, and the formation of autophagolysosomes, leading to an increase in intracellular levels of fatty acids. Inhibition of autophagy by bafilomycin A1 or chloroquine abolished the protection of apigenin in OA-induced lipid accumulation. Apigenin up-regulated the protein expression related to the $\beta$-oxidation pathway including acyl-CoA synthetase long chain family member 1, carnitine palmitoyltransferase $1 \alpha$, acyl-CoA oxidase 1, peroxisome proliferator activated receptor (PPAR) $\alpha$, and PPAR $\gamma$ coactivator 1- $\alpha$. Moreover, apigenin increased the mitochondrial network structure and mitochondrial function by increasing the protein expression related to the process of mitochondria fusion and mitochondrial function. Collectively, our findings suggest that apigenin ameliorates hepatic lipid accumulation by activating the autophagy-mitochondrial pathway.
\end{abstract}

Keywords: Apigenin, Autophagy, Hepatic lipid metabolism, Mitochondria

\section{Introduction}

A pigenin, a flavonoid component, is a natural constituent of many vegetables and fruits, especially parsley and celery [1,2]. During the past decade, considerable efforts have been made towards unveiling the potential of apigenin in treating many human diseases, particularly cancers, inflammatory diseases, and metabolic diseases [3-5]. For instance, apigenin induces cell death in human papillary thyroid carcinoma cells [6]. Apigenin exerts anti-inflammatory effects in acute pancreatitis by inhibiting the expression of tumor necrosis factor- $\alpha$ [7]. In addition, apigenin has the atheroprotective effect of increasing the cholesterol efflux in apoE-deficient mice [8]. Moreover, apigenin prevents high-fat diet (HFD)induced hyperlipidemia and non-alcoholic fatty

Abbreviations: apoE, apolipoprotein E; ATG, autophagy related protein; ATP, adenosine triphosphate; ACSL1, long-chain fatty acid coenzyme A ligase 1; ACOX1, acyl-coenzyme A oxidase 1; BafA1, bafilomycin A1; CPT1 $\alpha$, carnitine palmitoyltransferase 1; CQ, chloroquine; Drp1, dynamin-related protein 1; LC3, microtubule-associated protein 1A/1B-light chain 3; MTCO1, cytochrome c oxidase subunit 1; Mff, mitochondrial fission factor; Mfn2, mitofusin 2; MMP, mitochondrial membrane potential; NAFLD, non-alcoholic fatty liver disease; OA, oleic acid; OPA1, mitochondrial dynamin like GTPase; PPAR $\alpha$, peroxisome proliferator-activated receptor $\alpha$; PGC-1 $\alpha$, peroxisome proliferator-activated receptor $\gamma$ coactivator 1- $\alpha$; ROS, reactive oxygen species; SQSTM1, sequestosome 1.

Received 22 October 2020; revised 18 January 2021; accepted 22 February 2021

Available online 15 June 2021

* Corresponding author: Graduate Institute and Department of Physiology, College of Medicine, National Taiwan University, Taipei, 10051, Taiwan. Tel: +886-2-23123456 ext 88242; Fax: +886-2-2396-4350.

E-mail address: ntutslee@ntu.edu.tw (T.-S. Lee). 
liver disease (NAFLD) in mice [9]. Although the protective effects of apigenin on metabolic diseases have been studied extensively in vitro and in vivo [10], there is little information about the molecular mechanism underlying the protective effect of apigenin on the deregulation of lipid metabolism.

Autophagy is a conserved self-eating process that is important for the intracellular homeostasis of lipid metabolism upon stress conditions [11]. Autophagy is known to elicit the hydrolysis of triglycerides to fatty acids and attenuate the progression of NAFLD [12]. When the excess triglycerides are accumulated in the cells, the process of autophagy is initiated and triglycerides are encapsulated, and subsequently fused with the lysosome $[13,14]$. Lipids are broken down into fatty acids within the lysosome and then transported to mitochondria for $\beta$-oxidation and to produce adenosine triphosphate (ATP) [15]. However, whether apigenin induces autophagy and regulates the turnover of triglycerides to fatty acids is largely unknown. Nevertheless, the molecular mechanism behind the catabolic metabolism of lipid droplets to fatty acids contributing to apigenin-mediated protection in the lipid homeostasis of hepatocytes requires further investigation.

Mitochondria are the energy factories that provide the cell with energy by the biochemical processes of cellular respiration [16]. Disruption of this highly controlled process results in the deficiency in bioenergetics [17]. In response to different stimuli and metabolic demands of the cells, the number, shape, and structure of mitochondria are dynamically regulated by the balance of fusion and fission [18]. Indeed, growing evidence suggests that mitochondrial dysfunction is associated with metabolic diseases such as NAFLD [19,20]. The excess accumulation of lipids in hepatocytes creates the environmental cues and enhances the inputs of the electron transport chain, which in turn causes inefficient electron transport and results in the production of mitochondrial reactive oxygen species (ROS) [21,22]. Such metabolic insult-induced mitochondrial ROS disturbs the mitochondrial dynamics by deregulating the balance of fusion and fission, leading to the impairment of mitochondrial bioenergetics [23]. However, less is known about the effect of apigenin on the excess lipid-induced production of mitochondrial ROS and bioenergetics.

Given the beneficial effect of apigenin on lipid metabolism, we aimed to investigate the effect of apigenin as well as its molecular mechanism in oleic acid (OA)-induced lipid accumulation in hepatocytes.
We first investigated the effect of apigenin on OAinduced lipid accumulation and then examined whether apigenin affected the autophagy and the morphology and function of mitochondria in hepatocytes. Our findings suggest that treatment with apigenin induces autophagy, facilitates mitochondrial function, and results in an increase in the turnover of lipid droplets, ultimately leading to the amelioration of OA-induced lipid accumulation in the hepatocytes.

\section{Materials and methods}

\subsection{Reagents}

Apigenin, OA, acridine orange, bafilomycin A1 (BafA1), chloroquine (CQ), and oligomycin were from Cayman Chemical (Ann Arbor, MI, USA). Rabbit antibodies for Beclin1 (BECN1, sc-11427), long-chain fatty acid coenzyme A ligase 1 (ACSL1, sc-98925), and peroxisome proliferator-activated receptor $\alpha$ (PPAR $\alpha$, sc-9000) were obtained from Santa Cruz Biotechnology (Santa Cruz, CA, USA). Rabbit antibodies for microtubule-associated protein 1A/1B-light chain 3 (LC3, \#4108), sequestosome 1 (SQSTM1, \#5114), mitofusin 2 (Mfn2, \#9482), and dynamin-related protein 1 (Drp1, \#8570) were from Cell Signaling Technology (Beverly, MA, USA). Rotenone and rabbit antibodies for autophagy related 7 (ATG7, ab133528), acyl-coenzyme A oxidase 1 (ACOX1, ab59964), and peroxisome proliferator-activated receptor $\gamma$ coactivator $1-\alpha$ coactivator- $1 \alpha$ (PGC- $1 \alpha$, ab54481) and mouse antibodies for carnitine palmitoyltransferase $1 \alpha$ (CPT1 $\alpha$, ab128568) and cytochrome $\mathrm{c}$ oxidase subunit 1 (MTCO1, ab14705) were from Abcam (Cambridge, MA, UK). Rabbit antibody for autophagy related 5 (ATG5, nb110-53818) was from Novus Biologicals (Littleton, CO, USA). Rabbit antibody for mitochondrial fission factor (Mff, 17090-1-AP) was from Proteintech (Rosemont, IL, USA). Mouse antibody for mitochondrial dynamin like GTPase (OPA1, 612606) was from BD Bioscience (San Jose, CA, USA). Nile red and mouse antibody for $\alpha$-tubulin were from Sigma-Aldrich (St Louis, MO, USA). The colorimetric/fluorometric kit for free fatty acids was obtained from BioVision (Milpitas, CA, USA). JC-10 was from AAT Bioquest (Sunnyvale, CA, USA). MitoSOX Red and MitoTracker Green were all obtained from Invitrogen (Eugene, OR, USA).

\subsection{Cell culture}

Human hepatoma cells Huh7, Huh7.5 (stable LC3GFP-expressing), HepG2 and murine hepatocyte 
cell line AML12 were cultured in Dulbecco's modified Eagle's medium (DMEM) supplemented with $10 \%$ fetal bovine serum and penicillin $(100 \mathrm{U} / \mathrm{mL}) /$ streptomycin $(100 \mu \mathrm{g} / \mathrm{mL})$ in a humidified incubator at $37{ }^{\circ} \mathrm{C}, 95 \%$ air, and $5 \% \mathrm{CO}_{2}$.

\subsection{Lipid staining}

At the end of experiments, the confluence of Huh7 cells was $\sim 95 \%$. Intracellular lipid accumulation was assessed by oil red $\mathrm{O}$ and Nile red staining. Cells were fixed with $4 \%$ paraformaldehyde and then stained with $0.5 \%$ oil red $\mathrm{O}$ or Nile red $(1 \mu \mathrm{g} / \mathrm{mL})$ for $15 \mathrm{~min}$ at room temperature. Hematoxylin was used as a counterstain for oil red $\mathrm{O}$ staining. The fluorescence of Nile red was determined by fluorescence fluorometry (Molecular Devices, Sunnyvale, CA, USA) using 540-nm excitation and 590-nm emission wavelengths. The fluorescence was normalized to the total protein concentration. The fold of vehicle was defined as level of fluorescence intensity relative to the vehicle group set as 1 . Photomicrographs were digitally captured under a Leica DMIRB Microscope with LAS V4.12 software (Wetzlar, Germany).

\subsection{Western blot analysis}

Aliquots of cell lysates were separated on SDSPAGE and then transblotted onto the PVDF membrane (Millipore, Bedford, MA). After being blocked with $5 \%$ skim milk, the blotting membrane was then incubated with the primary antibodies, followed by the corresponding horseradish peroxidase-conjugated secondary antibodies. Bands were visualized by using an enzyme-linked chemiluminescence detection kit (PerkimElmer, Waltham, MA, USA), and the band density was measured using the TotalLab 1D (Newcastle Upon Tyne, UK).

\subsection{Visualization of acidic vesicular organelles}

Intracellular acidic vacuoles including lysosomes, endosomes, and autophagosomes were assessed by acridine orange staining. Living cells were stained by acridine orange $(2.7 \mu \mathrm{M})$ in culture medium for $15 \mathrm{~min}$ at $37^{\circ} \mathrm{C}$. The fluorescence of acridine orange staining was determined by fluorometry (Molecular Devices) using the excitation/emission spectra of $502 / 525 \mathrm{~nm}, 460 / 650 \mathrm{~nm}$, and 475/590 nm. Photomicrographs were digitally captured under a Leica DMIRB Microscope.

\subsection{The measurement of cellular free fatty acids}

The lipids in the cells were extracted by use of $n$ hexane/isopropanol $(3: 2, \mathrm{v} / \mathrm{v})$ for $1 \mathrm{~h}$ on a shaker. After drying, the extracted lipids were dissolved by using the commercial solutions, and the levels of intracellular free fatty acids were assessed by using fluorescence assay kits (BioVision, Milpitas, CA, USA). The fluorescence intensity of cell lysates was analyzed at $530 \mathrm{~nm}$ excitation and $590 \mathrm{~nm}$ emission wavelengths by SpectraMax i3x fluorometry (San Jose, CA, USA).

\subsection{Immunocytochemistry}

Cells were seeded into 4-well plates, incubated with the test reagents, and then fixed with $4 \%$ paraformaldehyde for $30 \mathrm{~min}$. After being permeabilized by $70 \%$ alcohol for $2 \mathrm{~h}$ at $4{ }^{\circ} \mathrm{C}$, cells were blocked in $2 \% \mathrm{BSA}$ for $30 \mathrm{~min}$ at $37^{\circ} \mathrm{C}$. Cells were incubated with primary antibody for $2 \mathrm{~h}$ at room temperature and then with the corresponding secondary antibody for overnight at $4{ }^{\circ} \mathrm{C}$. Photomicrographs were digitally captured under a Leica DMIRB Microscope.

\subsection{Mitochondrion-selective probes}

The detection of mitochondria was performed using MitoTracker reagent. Cells were incubated with medium containing MitoTracker reagent $(250 \mathrm{nM})$ at $37{ }^{\circ} \mathrm{C}$ for $30 \mathrm{~min}$. Photomicrographs were digitally captured under a Leica DMIRB Microscope.

\subsection{Mitochondrial superoxide indicator}

The production of mitochondria superoxide was measured by MitoSOX reagent. Cells were incubated with medium containing MitoSOX reagent $(5 \mu \mathrm{M})$ at $37{ }^{\circ} \mathrm{C}$ for $10 \mathrm{~min}$. The fluorescence of MitoSOX was determined by fluorometry (Molecular Devices) using the excitation/emission spectra of $510 / 580 \mathrm{~nm}$. Photomicrographs were digitally captured under a Leica DMIRB Microscope.

\subsection{Mitochondria membrane potential}

The membrane potential of mitochondria was assessed by mitochondria-specific probe JC- 10 . Cells were incubated with medium containing JC-10 $(3 \mu \mathrm{M})$ at $37{ }^{\circ} \mathrm{C}$ for $30 \mathrm{~min}$ and then stimulated 
A

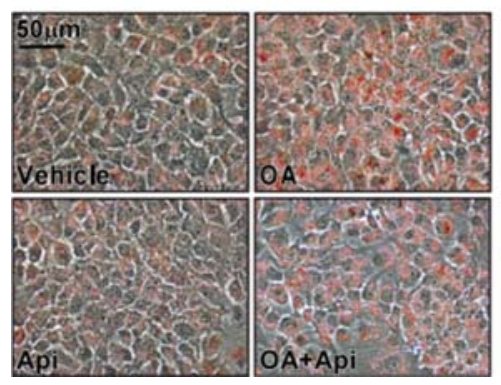

B

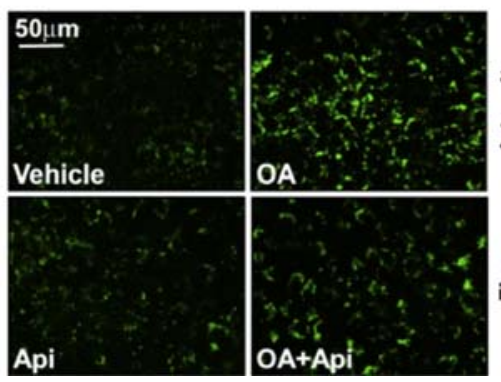

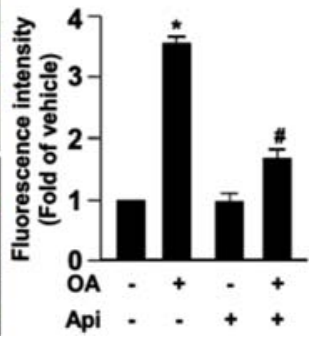

Fig. 1. Apigenin prevents oleic acid (OA)-induced lipid accumulation in Huh7 cells. Huh7 cells were pretreated with OA (125 $\mu \mathrm{g} / \mathrm{mL})$ for $24 \mathrm{~h}$ and then with or without apigenin $(20 \mu \mathrm{M})$ for an additional $18 \mathrm{~h}$. (A) Representative images of oil red O staining of Huh7 cells. (B) Representative images and the quantitative intensity of Nile red staining. Data are the mean \pm SEM from 5 independent experiments. $* P<0.05$ vs. the vehicletreated group; ${ }^{\# P}<0.05$ vs. the OA-treated group.

with test reagents for $10 \mathrm{~min}$. The fluorescence of JC-10 was determined by fluorometry (Molecular Devices) using the excitation/emission spectra of $490 / 525 \mathrm{~nm}$ and 540/590 nm. Photomicrographs were digitally captured under a Leica DMIRB Microscope.

\subsection{Mitochondrial bioenergetics}

The measurement of mitochondrial energy pathways was conducted according to the manufacturer's protocol by using the Seahorse XFe24 instrument and analyzed by software version Wave 2.2 (Seahorse Bioscience, Billerica, MA, USA).

\subsection{Statistical analysis}

The results are presented as the mean \pm SEM from 5 independent experiments and the sample size used for each statistical analysis is 5 . For comparisons of data of the two groups, the MannWhitney U test was used. For comparisons of data of more than two groups, one-way analysis of variance (ANOVA) followed by the Dunnett's multiple comparisons or Mann-Whitney U test was performed. SPSS software v8.0 (SPSS Inc., Chicago, IL) was used for all statistical analyses. Differences were considered statistically significant at $P<0.05$.

\section{Results}

\subsection{Apigenin prevents $O A$-induced lipid accumulation in $\mathrm{Huh} 7$ cells}

Previous studies indicate that excessive lipids accumulate in the hepatocytes during the development of NAFLD [12,19]. To clarify the potential effect of apigenin in regulating the lipid metabolism in hepatocytes, we used OA-induced intracellular lipid accumulation in Huh7 cells as our in vitro model. We first studied the cytotoxic effect of apigenin on the cell viability. The exposure of Huh7 cells to various concentrations $(0,2.5,5,10,20,40,80$, and $160 \mu \mathrm{M})$ of apigenin for $18 \mathrm{~h}$ and the results of the MTT assay showed that apigenin had no cytotoxic effect on cell viability at the concentrations ranging from 2.5 to $20 \mu \mathrm{M}$. However, apigenin induced cell death at concentrations starting from $40 \mu \mathrm{M}$ (Supplementary Fig. S1A). Thus, $20 \mu \mathrm{M}$ apigenin was chosen as the standard exposure for subsequent studies. Oil red $\mathrm{O}$ and Nile red staining showed that the intracellular levels of lipids in Huh7 cells were increased in response to OA challenge (Fig. 1A and B). Importantly, the OA-induced increase in intracellular lipid accumulation was abolished by the treatment with apigenin (Fig. 1A and B). In addition, such an inhibitory effect on lipid accumulation was observed in HepG2 and AML12 cells (Supplementary Fig. S2 and S3). These findings suggest that apigenin has a protective effect on lipid metabolism of hepatocytes.

\subsection{The activation of autophagy flux is required for the apigenin-conferred protection in $\mathrm{OA}$-induced deregulation of lipid metabolism}

Autophagy is known to play a crucial role in the degradation of intracellular lipid droplets [11,12]. We then examined the effect of apigenin on autophagy and found that exposure of OA-treated Huh7 cells to apigenin induced an increase in the expression of autophagy pathway-related proteins including Beclin 1, ATG5, ATG7, LC3-I, LC-3-II, and SQSTM1 in a concentration-dependent manner (Fig. 2A-F). Additionally, treatment with apigenin time-dependently induced the formation of autophagosomes as evidenced by the appearance of 
A

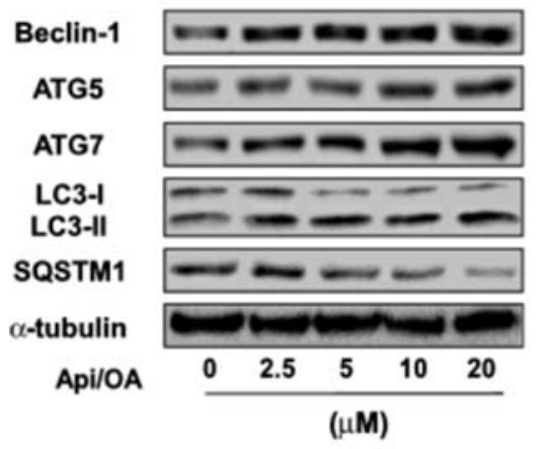

D

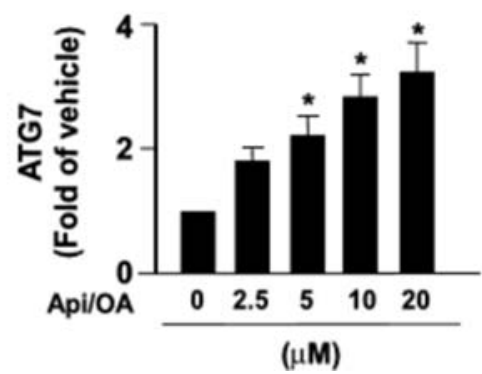

B

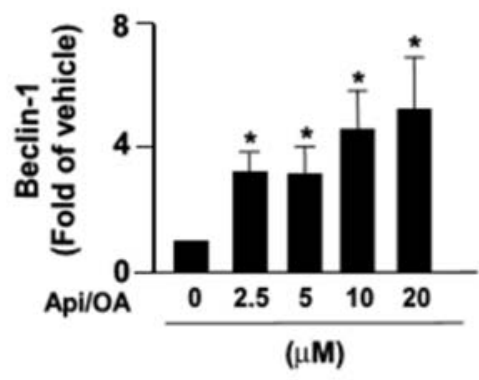

E

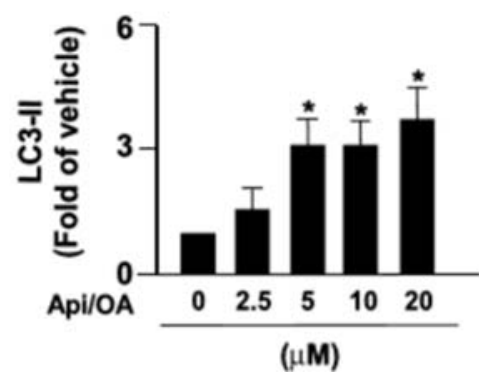

C

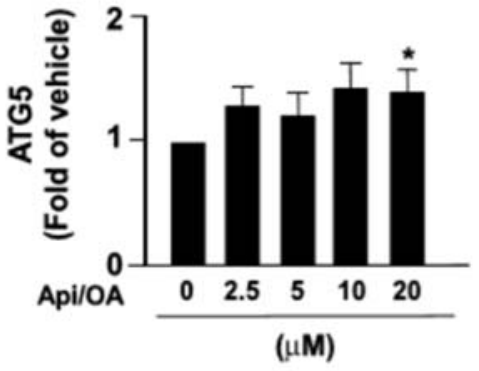

$\mathbf{F}$

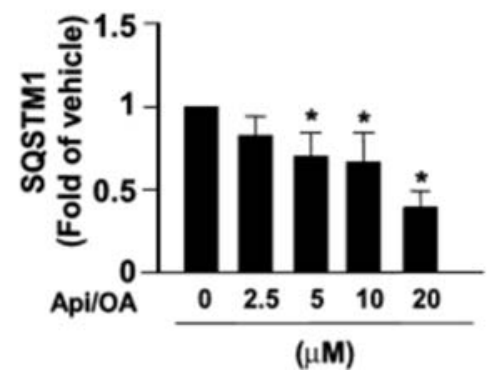

Fig. 2. Apigenin induces autophagy in Huh7 cells. (A-F) Huh7 cells were pretreated with $O A$ (125 $\mu \mathrm{g} / \mathrm{mL}$ ) for $24 \mathrm{~h}$ and then apigenin (20 $\mu \mathrm{M})$ for the indicated concentrations. Western blot analysis of protein levels of Beclin1, ATG5, ATG7, LC3, SQSTM1, and $\alpha$-tubulin. Data are the mean $\pm S E M$ from 5 independent experiments. ${ }^{*} P<0.05$ vs. the $0 \mu M$ group.

GFP-LC3 puncta as early as $6 \mathrm{~h}$ after treatment (Fig. 3A). Moreover, the results of acridine orange staining showed that apigenin time-dependently increased the autolysosome lysosomal activity as evidence by the increased intracellular levels of acidic vacuoles within $6-9 \mathrm{~h}$ after treatment (Fig. 3B). Finally, the intracellular levels of free fatty acids were increased after apigenin treatment (Fig. 3C). We next assessed whether the activation of autophagy was involved in the beneficial effect of apigenin on OA-induced lipid accumulation in hepatocytes. Pretreatment with BafA1 or CQ, two autophagy inhibitors for blocking the formation of autolysosome, abolished that protective effect of apigenin on the OA-induced lipid accumulation (Fig. 4A-D). Collectively, these findings suggest the activation of the autophagy pathway is essential for the beneficial effect of apigenin on the degradation of lipid droplets induced by OA in hepatocytes.

\subsection{Apigenin activates the $\beta$-oxidation of free fatty acids in Huh7 cells}

The activity of $\beta$-oxidation of free fatty acids in mitochondria plays a central role for maintaining the homeostasis of energy metabolism [16,19]. Inhibition of fatty acid oxidation damages energy metabolism and accelerates the development of NAFLD $[16,19]$. We next investigated the effect of apigenin on the $\beta$-oxidation pathway; we found that apigenin concentration-dependently increased the expression of $\beta$-oxidation pathway-related proteins including ACSL1, CTP1 $\alpha$, ACOX1, PPAR $\alpha$, and PGC1 $\alpha$ in OA-treated Huh7 cells (Fig. 5A-F). These findings suggest that apigenin may promote the activity of $\beta$-oxidation of free fatty acids to reduce the intracellular levels of lipids.

\subsection{Apigenin rescues the $O A$-induced mitochondrial dysfunctions in Huh7 cells}

We next investigated whether the increased activity of $\beta$-oxidation by apigenin was attributed to the altered mitochondrial dynamics. Our data revealed that apigenin prevented the OA-induced increase in the number of mitochondria as evidenced by the expression of MTCO1, a key enzyme in the mitochondrial electron transport chain (Fig. 6A and B). In addition, apigenin up-regulated the expression of mitochondrial fusion-related proteins, such as OPA1 and Mfn2; however, it had no effect on the expression of fission-related proteins, including Mff and Drp1 (Fig. 6C-G). Moreover, the results of fluorescent microscopy showed that $\mathrm{OA}$ 
A
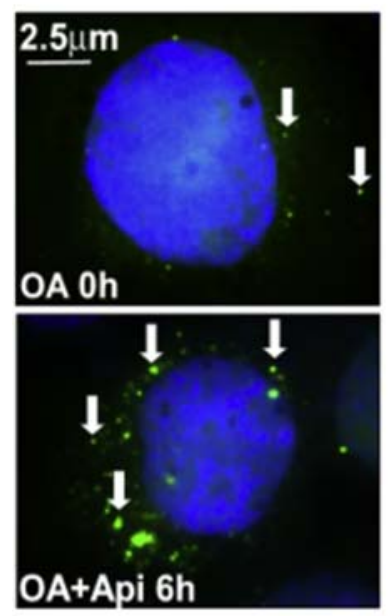
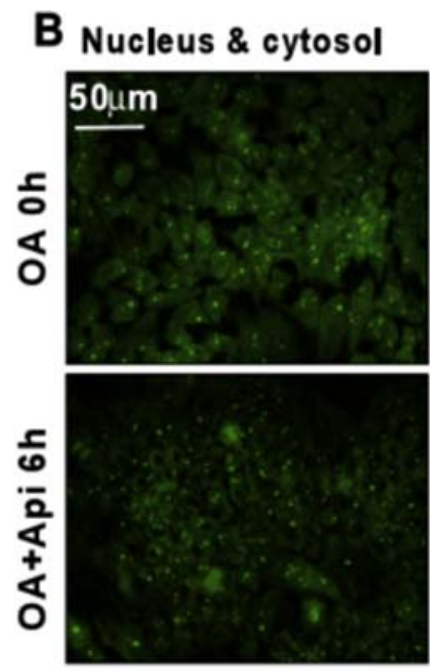

Acidic vacuoles
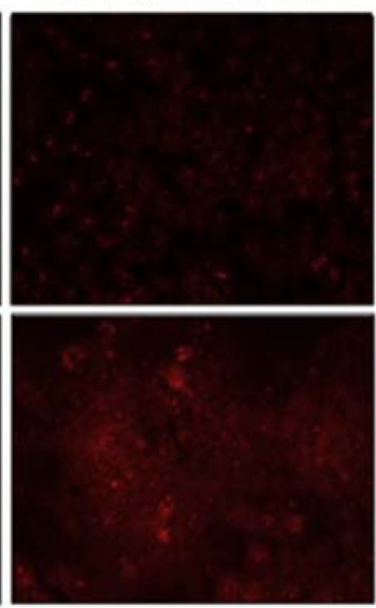

erged
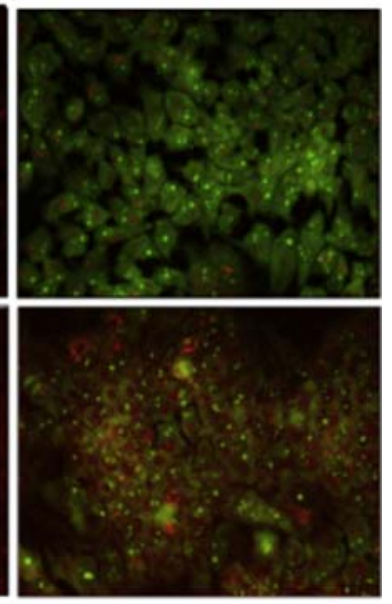

Acridine orange staining

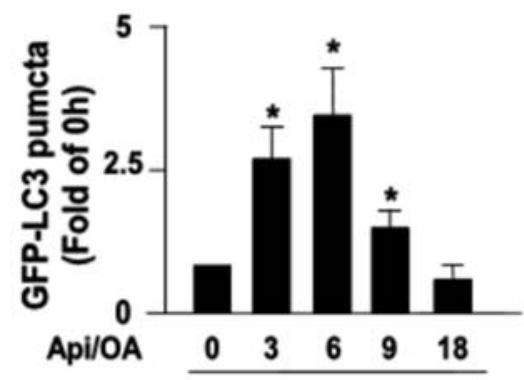

Time (h)

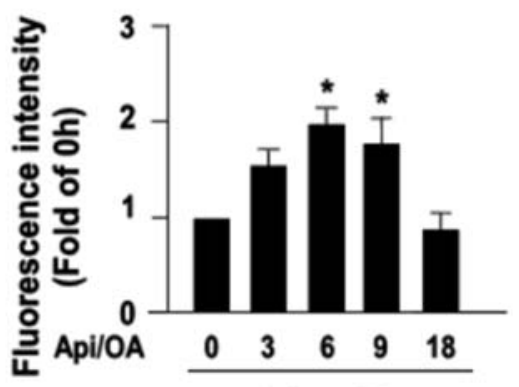

Time (h)

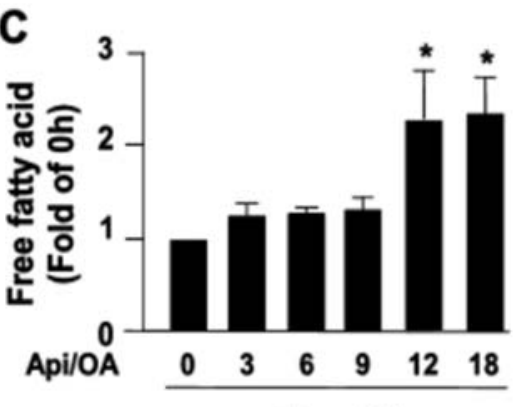

Time (h)

Fig. 3. Apigenin induces the formation of GFP-LC3 puncta and activates lysosome-dependent autophagy pathway. Huh7.5 cells were pretreated with $\mathrm{OA}(125 \mu \mathrm{g} / \mathrm{mL})$ for $24 \mathrm{~h}$ and then apigenin $(20 \mu \mathrm{M})$ for the indicated times. (A) Representative images and the quantitative result of GFP-LC3 puncta. (B) Representative images, acidic vacuoles (red fluorescence), the nucleus and cytoplasm (green fluorescence), and the quantitative intensity of acridine orange staining of Huh7 cells. (C) The intracellular levels of free fatty acids in Huh7 cells with apigenin (20 $\mu M)$ for indicated times. Data are the mean \pm SEM from 5 independent experiments. $* P<0.05$ vs. the 0 h group.

inhibited the mitochondrial fusion as evidenced by an increase in fragmented round forms of mitochondria; in contrast, apigenin promoted mitochondrial fusion as evidenced by an increase in elongated tubular forms of mitochondria and prevented the OA-induced inhibition of mitochondrial fusion (Fig. 6H).

Increased levels of mitochondrial ROS deregulate the mitochondrial dynamics resulting in the impairment of mitochondrial membrane potential (MMP) and mitochondrial function [23]. We then explored the effect of apigenin on the production of mitochondrial ROS elicited by OA. Our results revealed that treatment with apigenin abolished the OA-induced increase in the production of mitochondrial ROS (Fig. 7) and prevented the OAinduced damage in MMP (Fig. 8). Furthermore, the results of Seahorse XFe24 analysis demonstrated that treatment with apigenin prevented the detrimental effect of $\mathrm{OA}$ on mitochondrial function including cellular basal respiration, ATP production, maximal respiration, and proton leak, all of which were abolished by treatment with BafA1 or $\mathrm{CQ}$ in hepatocytes (Fig. 9A-E). To ensure the involvement of mitochondrial function in the apigenin-mediated protection in lipid metabolism, Huh7 cells were pretreated with oligomycin or rotenone, the specific inhibitor for the ATPase and mitochondrial complex I, respectively, and the results showed that inhibition of mitochondrial function diminished the apigenin-conferred protection on OA-induced lipid accumulation in Huh7 cells (Fig. 10A-D). Collectively, these results indicate that apigenin has a protective effect against OAinduced mitochondrial dysfunction.

\section{Discussion}

Apigenin is known to have protective effects against metabolic diseases [5,24]; however, the 

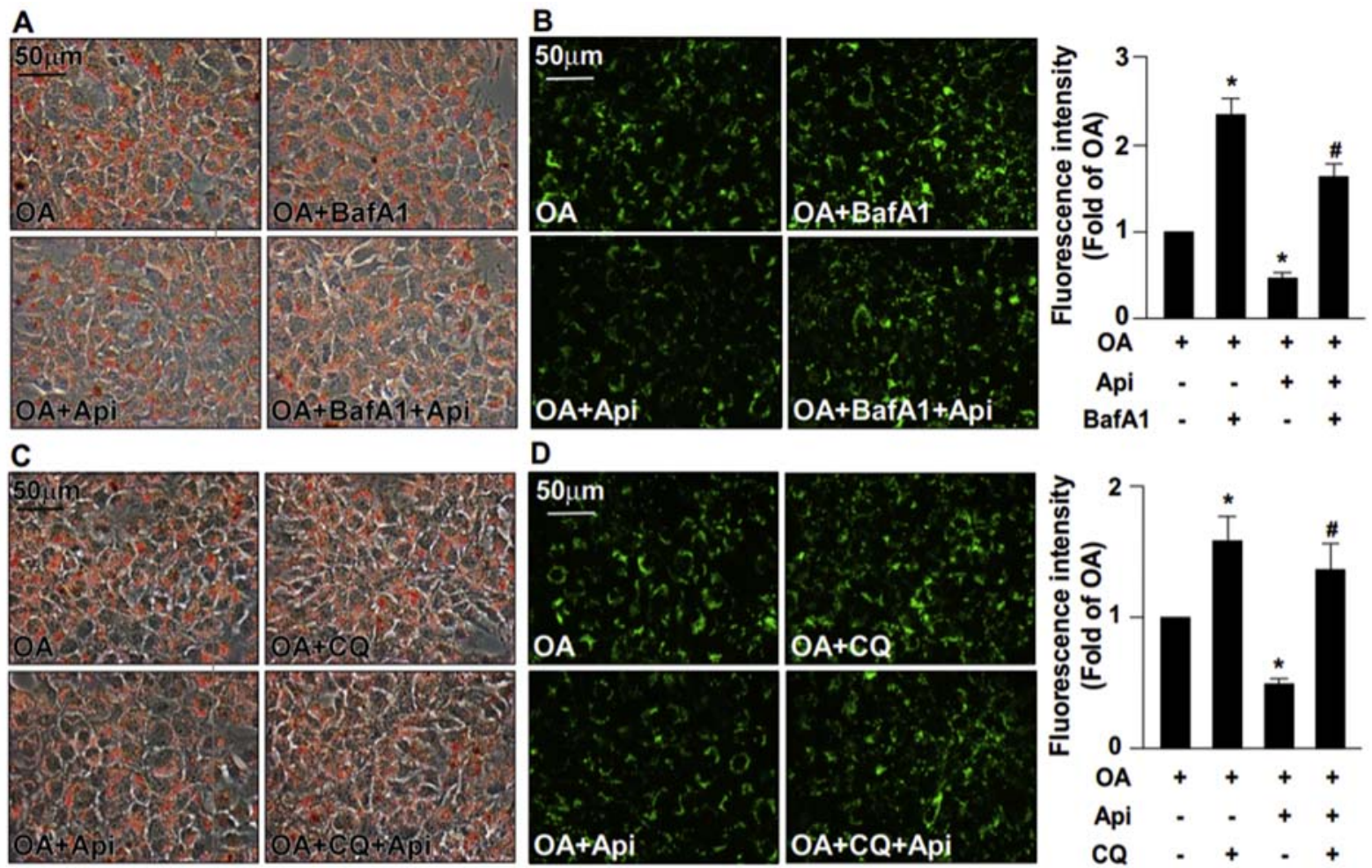

Fig. 4. Inhibition of autophagy abolishes the apigenin-mediated protection from OA-induced lipid accumulation. Huh7 cells were pretreated with $O A(125 \mu \mathrm{g} / \mathrm{mL})$ for $24 \mathrm{~h}$ and then with or without autophagy inhibitors (bafilomycin A1, BafA1, $100 \mathrm{nM}$, or chloroquine, CQ, $40 \mu \mathrm{M})$ for $2 \mathrm{~h}$, followed by apigenin $(20 \mu \mathrm{M})$ for an additional $18 \mathrm{~h}$. (A and $C)$ Representative image of oil red $O$ staining. (B and $D)$ The representative images and the quantitative intensity of Nile red staining. Data are the mean $\pm S E M$ from 5 independent experiments. ${ }^{*} P<0.05$ vs. the OA-treated group; ${ }^{\#} P<0.05$ vs. the $O A+$ apigenin-treated group.

A

D
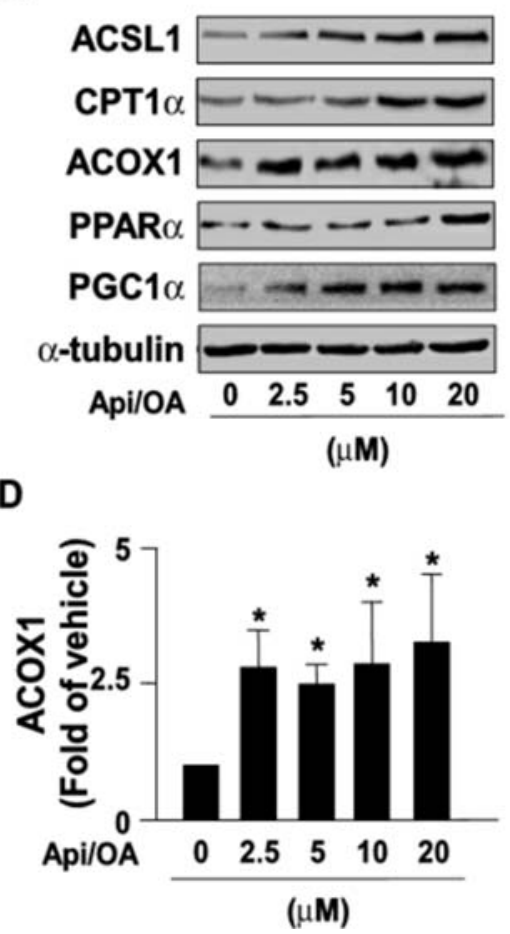

B

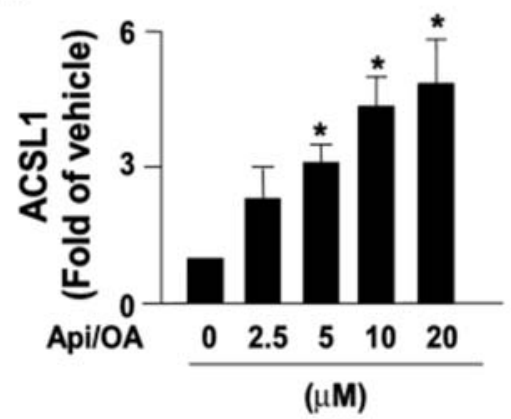

E

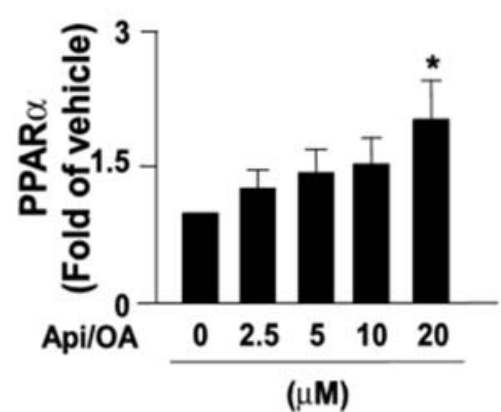

C

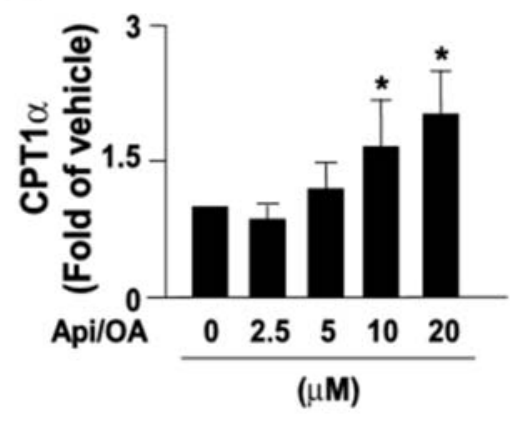

$\mathbf{F}$

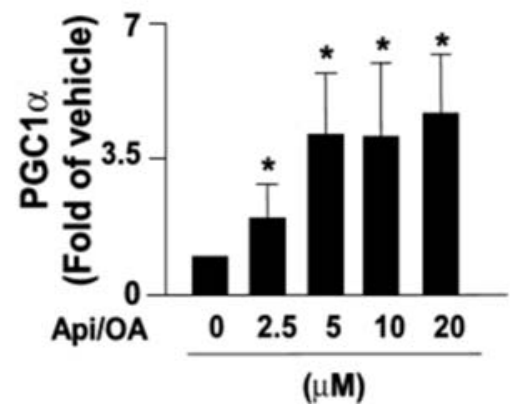

Fig. 5. Apigenin activates the $\beta$-oxidation pathway. (A-F) Huh7 cells were pretreated with $O A$ (125 $\mu g / m L)$ for 24 h and then apigenin (20 $\mu M$ ) for the indicated concentrations. Western blot analysis of protein levels of ACSL1, CPT1 $\alpha$, ACOX1, PPAR $\alpha, P G C 1 \alpha$, and $\alpha$-tubulin. Data are the mean $\pm S E M$ from 5 independent experiments. ${ }^{*} P<0.05 \mathrm{vs}$. the $0 \mu M$ group. 
A

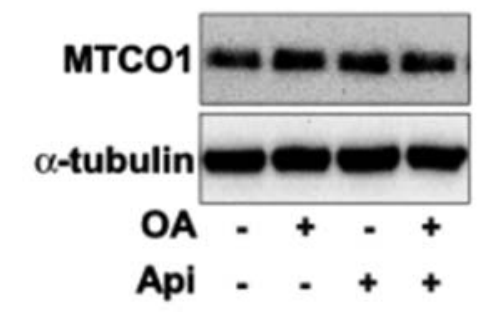

B

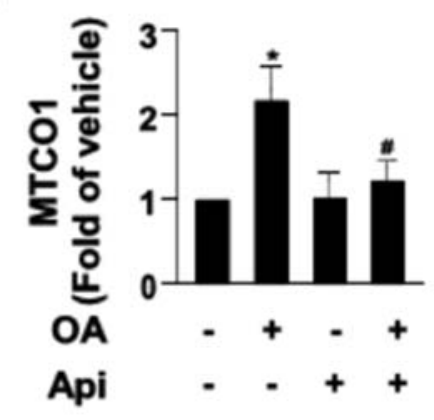

C

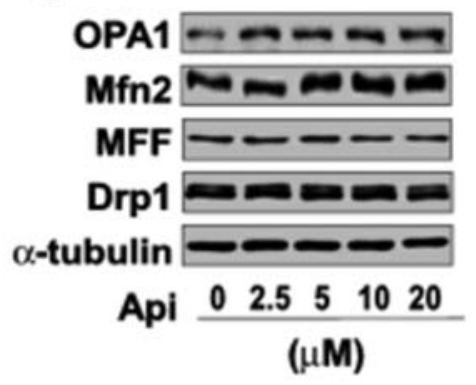

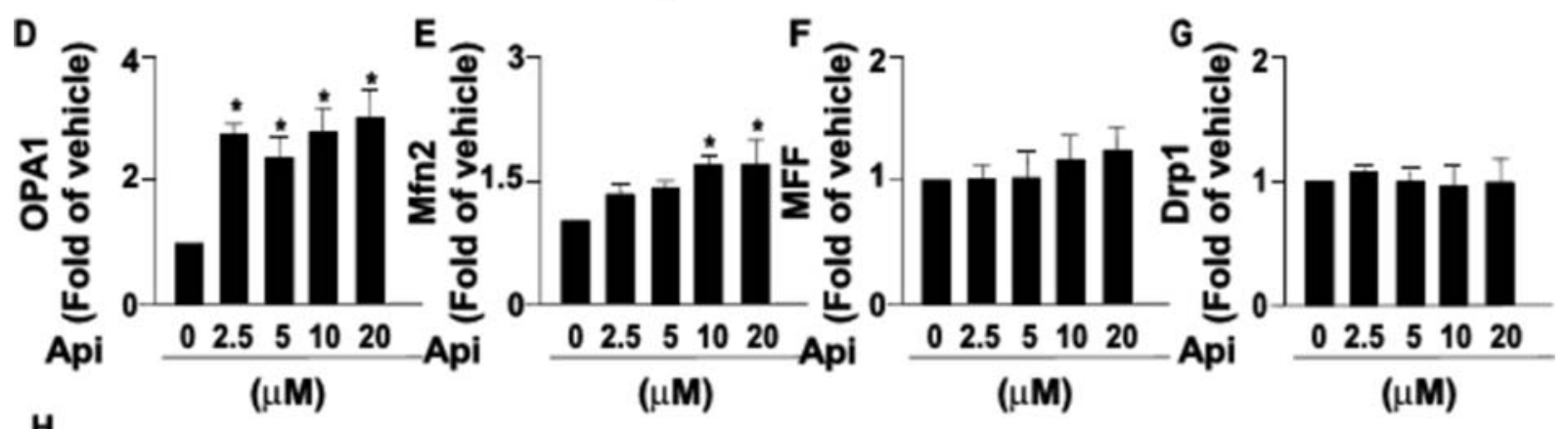

H
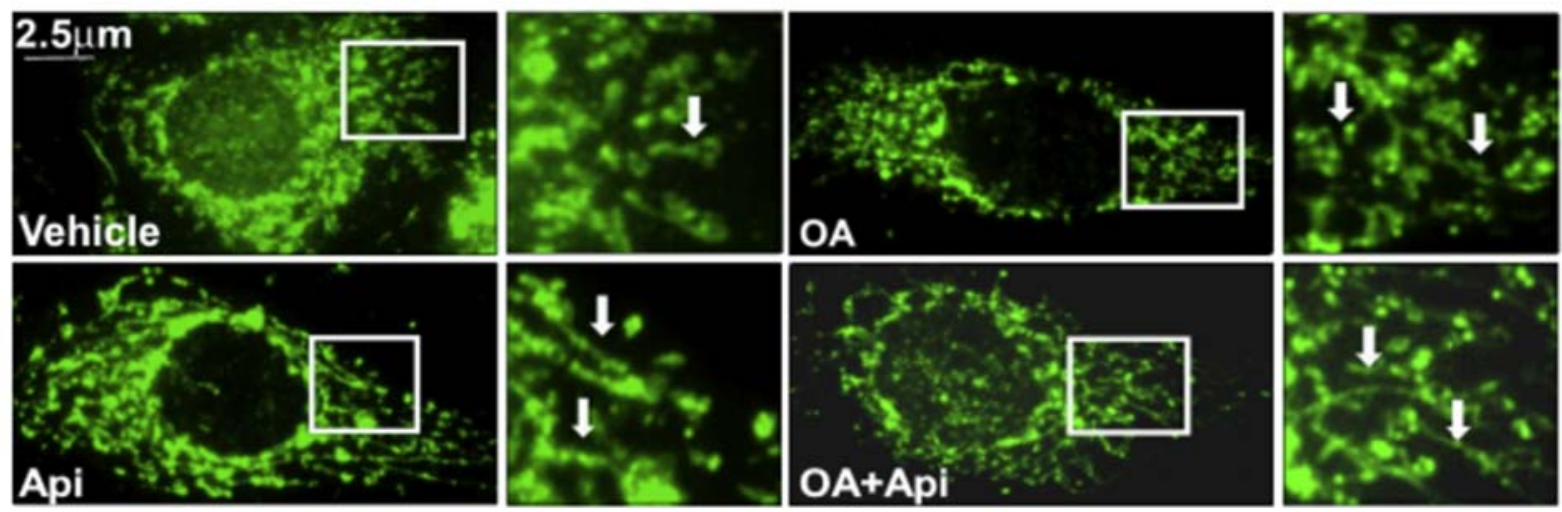

Fig. 6. Effect of apigenin on the mitochondrial biogenesis and dynamics in hepatocytes. (A and B) Huh7 cells were treated with OA (125 $\mu \mathrm{g} / \mathrm{mL}$ ) for $24 \mathrm{~h}$ in the absence or presence of apigenin $(20 \mu \mathrm{M})$. Western blot analysis of protein levels of MTCO1 and $\alpha$-tubulin. $(C-G) H u h 7$ cells were treated with apigenin $(20 \mu \mathrm{M})$ for the indicated concentrations. Western blot analysis of protein levels of OPA1, Mfn2, Mff, Drp1, and $\alpha$-tubulin. (H) Representative images of mitochondrial morphologies as indicated by arrows. Data are the mean \pm SEM from 5 independent experiments. $* P<0.05$ vs. the vehicle and $0 \mu M$ group.

molecular mechanism underlying the protective effect of apigenin on the deregulation of lipid metabolism has not yet been fully investigated. In this study, we used OA-induced lipid accumulation as our in vitro model and characterized a potential mechanism for apigenin in decreasing the lipid accumulation in hepatocytes. In Huh7 cells, exposure to excess OA induced the deregulation of lipid metabolism, which was attenuated by apigenin. Mechanistically, apigenin elicited the autophagy flux and increased the $\beta$-oxidation of fatty acids and mitochondrial bioenergetics and ultimately decreased the lipid accumulation. Additionally, apigenin promoted mitochondrial function by reducing the production of mitochondrial ROS and regulating its biogenesis, dynamics, and membrane potential. Ultimately, apigenin accelerated the conversion the lipids into energy to maintain the metabolic balance of hepatocytes (Fig. 11). Our findings regarding the benefits of apigenin in activating the autophagymitochondria pathway were in agreement with the previous reports that increasing the function of autophagy and mitochondria has therapeutic value in treating lipid metabolism disorders and related metabolic diseases $[25,26]$. Based on these observations in this study, we provided new insights into the protective effect and the underlying molecular mechanisms of apigenin in hepatic lipid metabolism. 

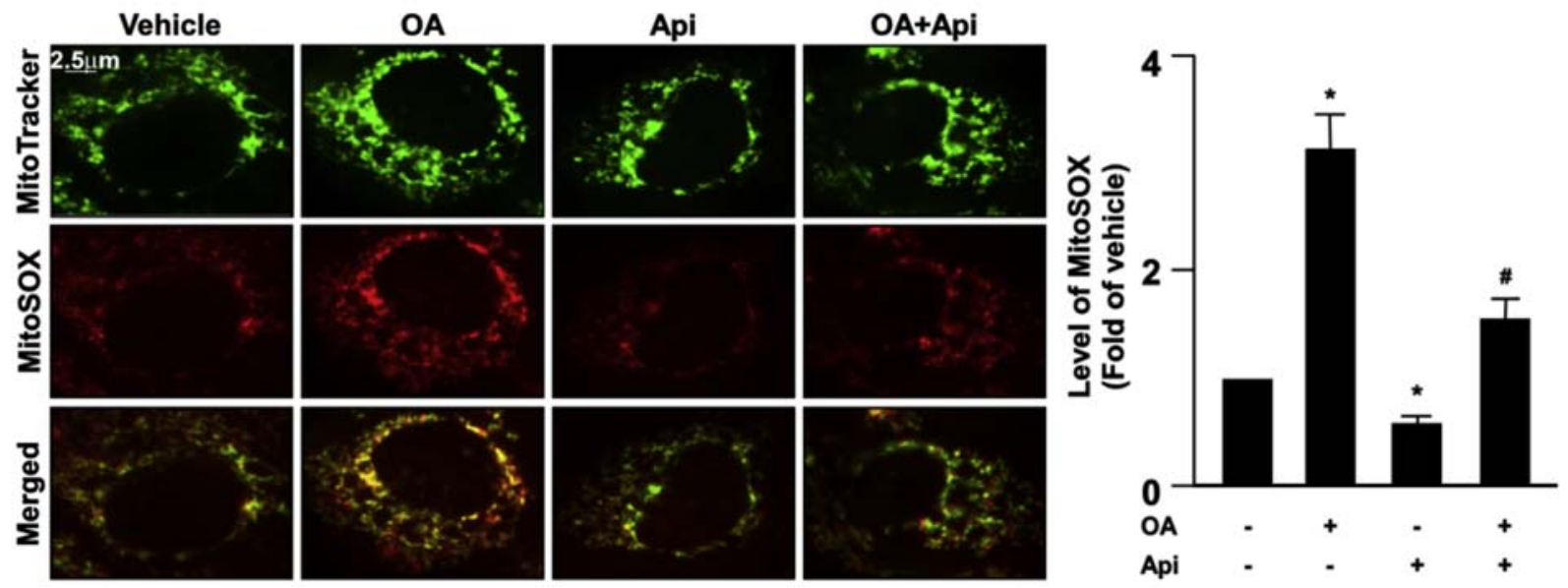

Fig. 7. Apigenin prevents $\mathrm{OA}$-induced mitochondrial superoxide production in hepatocytes. Huh7 cells were pretreated with $\mathrm{OA}$ (125 $\mu \mathrm{g} / \mathrm{mL}$ ) for $24 \mathrm{~h}$ and then apigenin $(20 \mu \mathrm{M})$ for $18 \mathrm{~h}$. The MitoTracker and MitoSOX assay kits were used to measurement of the mitochondrial superoxide production. Data are the mean \pm SEM from 5 independent experiments. ${ }^{*} P<0.05$ vs. the vehicle-treated group; ${ }^{\#}<0.05$ vs. the OA-treated group.

Interestingly, Chen et al. reported that apigenin elicits the activation of autophagy and induces programmed cell death, leading to the inhibition of tumor growth [27], which is in line with our finding that autophagy plays a crucial role in the beneficial effect of apigenin on lipid metabolism. However, to our knowledge, the detailed molecular mechanism behind the protective effect of apigenin on autophagy flux is not fully understood. Importantly, AMP-activated protein kinase (AMPK) is known to be a key player in regulating autophagy and lipid metabolism [28,29]; AMPK positively regulates the initiation of autophagy and promotes the clearance of intracellular lipid droplets [30]. Activation of AMPK signaling by pharmacological activators increases autophagy and leads to the decrease in the lipid accumulation in the liver [31]. Moreover, Lu et al. suggested that apigenin activates the AMPK signaling pathway and improves the deregulation of lipid metabolism, leading to the alleviation of lipid accumulation in HepG2 cells [32]. In contrast, the mammalian target of rapamycin (mTOR), a wellknown inhibitor for autophagy, plays a central role in the deregulation of lipid metabolism and development of NAFLD [33]. Zhou and Ye reported that the inhibition of the mTOR pathway by rapamycin increases autophagy and attenuates hepatic steatosis in rats with type 2 diabetes [34]. Recently, Farah et al. reported that pharmacological induction of autophagy by rapamycin improves glucose-6phosphatase deficiency-induced hepatic lipid metabolism in mice [35]. Nevertheless, whether the AMPK pathway or the mTOR signaling involved in the beneficial effect of apigenin on lipid metabolism

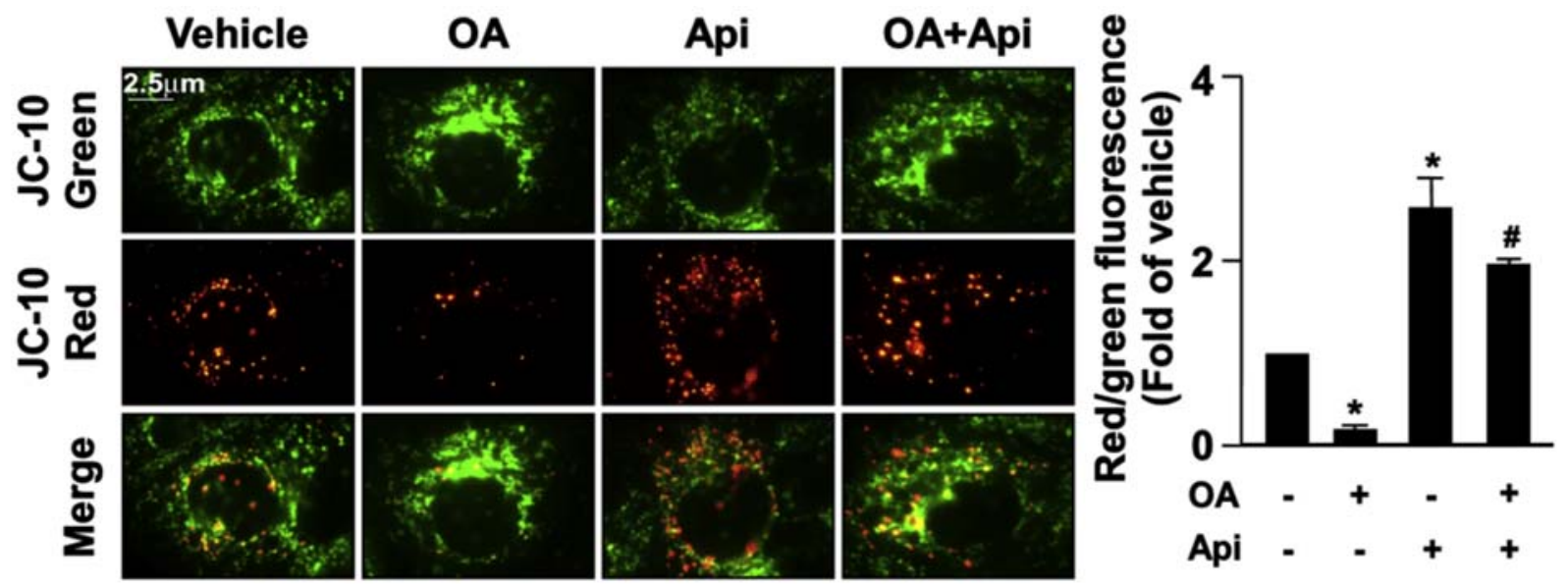

Fig. 8. Apigenin prevents the OA-induced damage on mitochondrial membrane potential in hepatocytes. Huh7 cells were pretreated with OA $(125 \mu \mathrm{g} / \mathrm{mL})$ for $24 \mathrm{~h}$ and then apigenin $(20 \mu \mathrm{M})$ for $18 \mathrm{~h}$. The JC-10 assay was used to detect the mitochondrial membrane potential. Data are the mean \pm SEM from 5 independent experiments. ${ }^{*} P<0.05$ vs. the vehicle-treated group; ${ }^{\#} P<0.05$ vs. the OA-treated group. 
in hepatocytes is unclear. To this end, further investigations for clarifying the molecular mechanism underlying the protective effect of apigenin on lipid metabolism are warranted.

Maintenance of mitochondrial function is a key event in regulating the normal function of hepatic lipid metabolism [36]. It is known that dynamic regulation in mitochondrial biogenesis, morphological changes, ROS production, and membrane potential are important for maintaining mitochondrial hemostasis [37-40]. It is well-established that mitochondrial dysfunction is highly associated with metabolic diseases, including NAFLD, hyperlipidemia, and atherosclerosis [20,41]. Disturbance of mitochondrial function induces the deregulation of lipid metabolism and accelerates the progression of metabolic diseases $[19,41]$. Rescuing the mitochondrial dysfunction by pharmacological reagents or supplementation with nutrients in foods alleviates the progression of metabolic diseases [42,43], suggesting that improving the mitochondrial dysfunction has therapeutic value in treating metabolic diseases. Interestingly, Choi et al. demonstrated that apigenin ameliorates obesity-induced skeletal muscle atrophy by attenuating mitochondrial dysfunction in vivo and in vitro [44]. The notion was further supported by our findings that apigenin decreases the excessive fatty acid-induced lipid accumulation in hepatocytes by increasing the mitochondrial biogenesis and fusion, decreasing mitochondrial ROS production, and correcting the deregulation of membrane potential of mitochondria. Intriguingly, we found that $\mathrm{OA}$ also increased mitochondrial biogenesis in our in vitro model, which is in line with the results of Rehman et al., who found that OA at a low dose could elicit the biogenesis of mitochondria against the diabetic insults in endothelial cells [45]. Therefore, we thought that increased mitochondrial biogenesis by $\mathrm{OA}$ in this study was a compensatory protective response for the deregulation of lipid metabolism in hepatocytes. In addition to the beneficial effect on mitochondrial functions, our data demonstrated that apigenin increased the expression of proteins
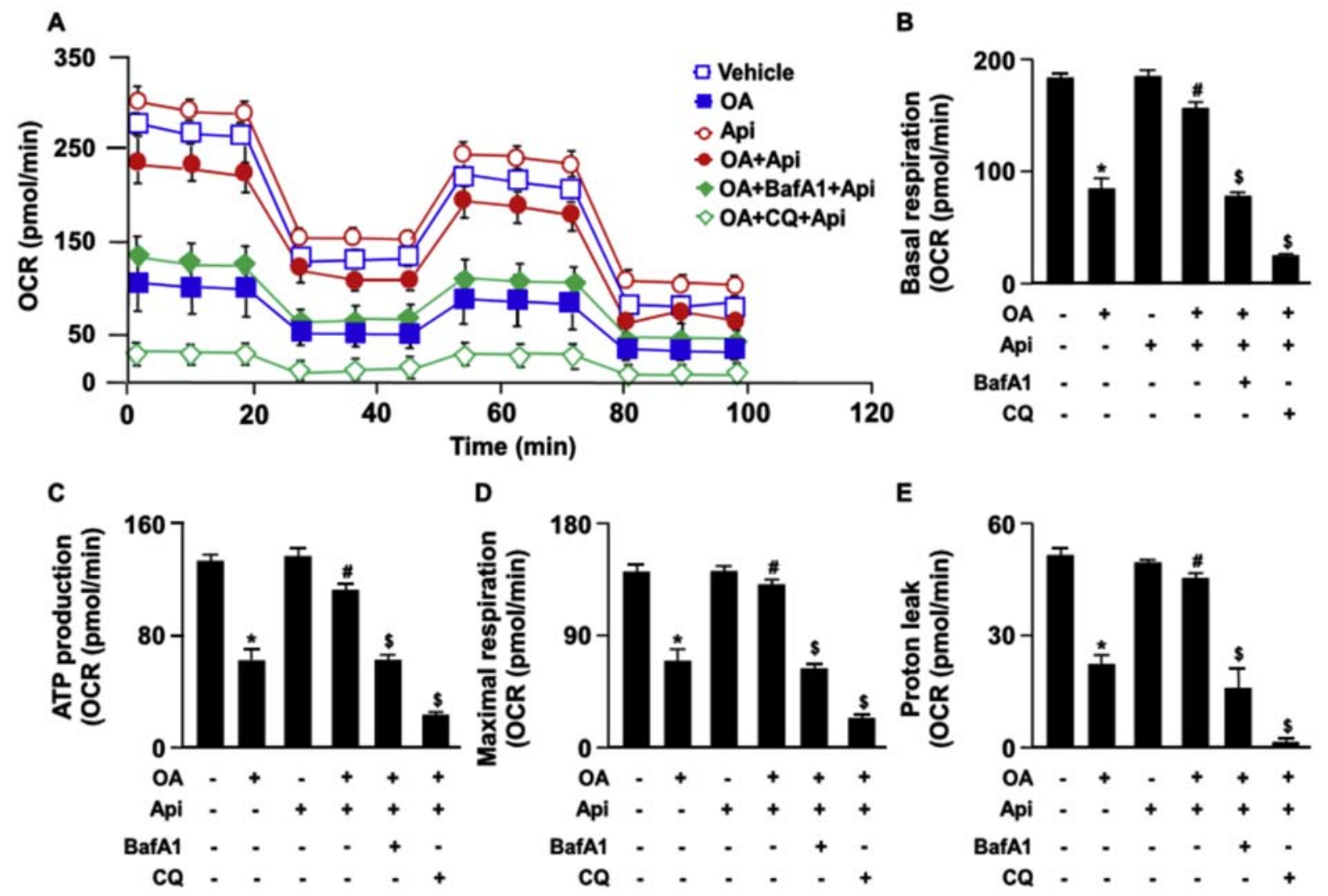

E

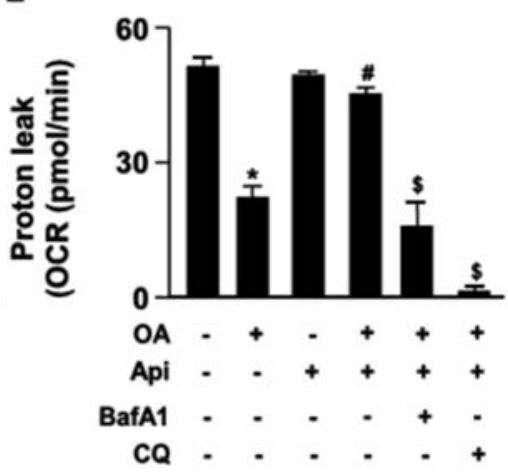

Fig. 9. Apigenin rescues the OA-induced mitochondrial dysfunction in hepatocytes. Huh7 cells were pretreated with $\mathrm{OA}(125 \mu \mathrm{g} / \mathrm{mL})$ for $24 \mathrm{~h}$ and then with or without autophagy inhibitors (bafilomycin A1, BafA1, $100 \mathrm{nM}$, or chloroquine, CQ, $40 \mu \mathrm{M})$ for $2 \mathrm{~h}$, followed by apigenin (20 $\mu \mathrm{M}$ ) for an additional $18 \mathrm{~h}$. (A-E) The Seahorse XFe24 instrument was used for the measurement of mitochondrial energy pathways including the cellular basal respiration, ATP production, maximal respiration, and proton leak. Data are the mean \pm SEM from 5 independent experiments. ${ }^{*} P<0.05$ vs. the vehicle-treated group; ${ }^{\# P}<0.05$ vs. the OA-treated group; ${ }^{\$} \mathrm{P}<0.05$ vs. the $\mathrm{OA}+$ apigenin-treated group. 
A

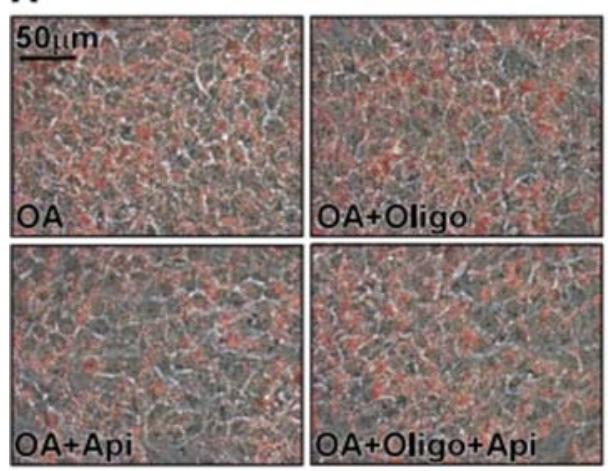

C
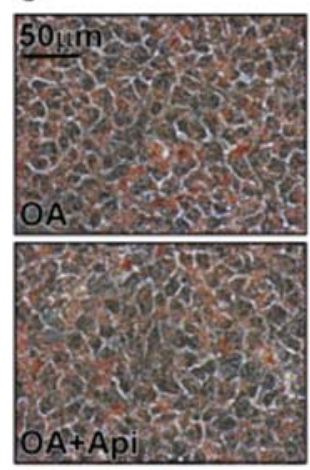
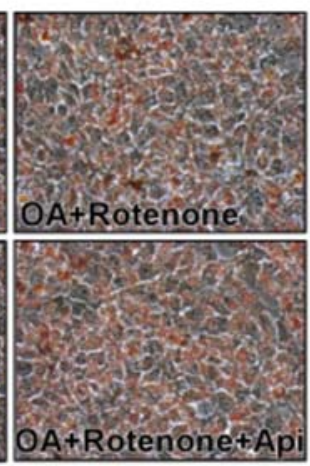

B
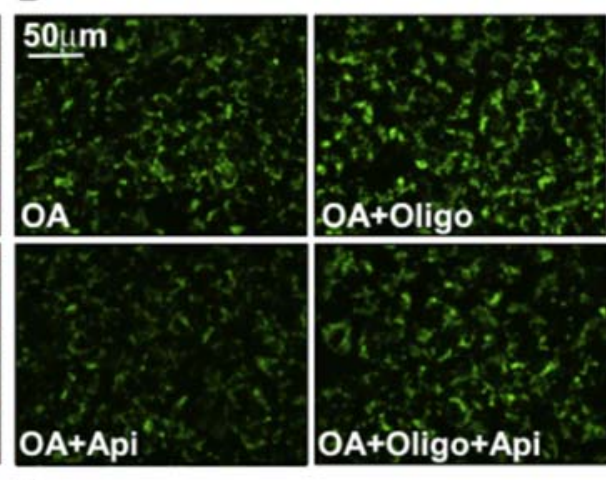

D
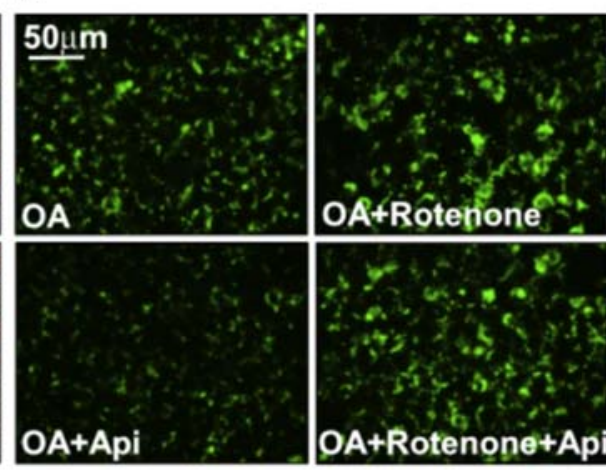
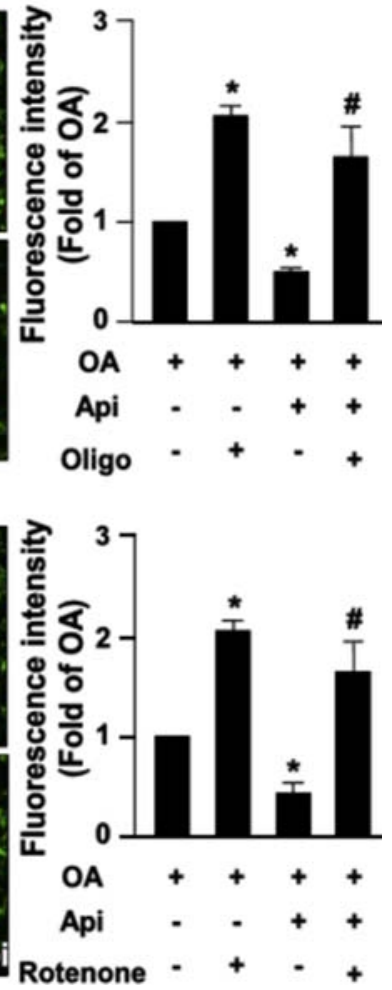

Fig. 10. Inhibition of mitochondrial function diminishes the apigenin-conferred protection from OA-induced lipid accumulation. Huh7 cells were pretreated with $\mathrm{OA}(125 \mu \mathrm{g} / \mathrm{mL})$ for $24 \mathrm{~h}$, autophagy inhibitor with or without mitochondrial inhibitors (oligomycin, Oligo, $0.5 \mu M$, or Rotenone, $0.5 \mu M)$ for $2 h$, and then with or without apigenin $(20 \mu M)$ for an additional $18 \mathrm{~h}$. (A and C) Representative images of oil red $O$ staining of Huh7 cells. (B and D) Representative images of the intracellular lipid accumulation and thee quantitative results of Nile red staining. Data are the mean \pm SEM from five independent experiments. ${ }^{*} P<0.05$ vs. the OA-treated group; ${ }^{\#} P<0.05$ vs. the OA + apigenin-treated group.

related to the $\beta$-oxidation of free fatty acids, which is in agreement with the previous findings that supplementation with flavonoids promoted free fatty acid oxidation and protection from metabolic diseases [46]. Collectively, these observations suggest that apigenin prevents excess fatty acid-induced mitochondrial dysfunction and promotes the $\beta$ oxidation of free fatty acids, and these two events may work in concert to decrease the lipid accumulation in hepatocytes.

ROS is an important secondary messenger for regulating cellular physiological functions and the development of metabolic diseases [47]. In the initial stage of metabolic diseases, ROS-mediated oxidative stress disturbs intracellular signaling cascades, deregulates physiological functions, and leads to the progression of metabolic disease [48]. Mitochondrial ROS is mainly generated by the electron transport chain located on the inner mitochondrial membrane during the process of oxidative phosphorylation and is involved in the regulation of lipid metabolism, inflammatory response, and apoptosis/autophagy pathways [49,50]. Increasing lines of evidence suggest that targeting the ROS pathway with antioxidants has been considered as an effective therapeutic strategy for preventing oxidative stressmediated metabolic events in clinical trials and animal studies [51]. Recently, Feng et al. reported that apigenin ameliorates HFD-induced NAFLD by modulating the oxidative stress in the liver [10]. Moreover, Zhong et al. demonstrated that apigenin attenuates apoptosis by decreasing ROS-mediated mitochondrial dysfunction [52]. This notion is further supported by our findings that treatment with apigenin abrogated the OA-induced lipid accumulation by decreasing the production of mitochondrial ROS in Huh7 cells. Conceivably, these findings suggest that the antioxidative property of apigenin may have therapeutic value in the treatment of metabolic diseases. Nonetheless, determining whether apigenin prevents the ROSmediated detrimental effects on metabolic diseases and its underlying molecular mechanism requires further investigations and clinical trials.

Our study contains several limitations because we did not use animal models to mimic the 


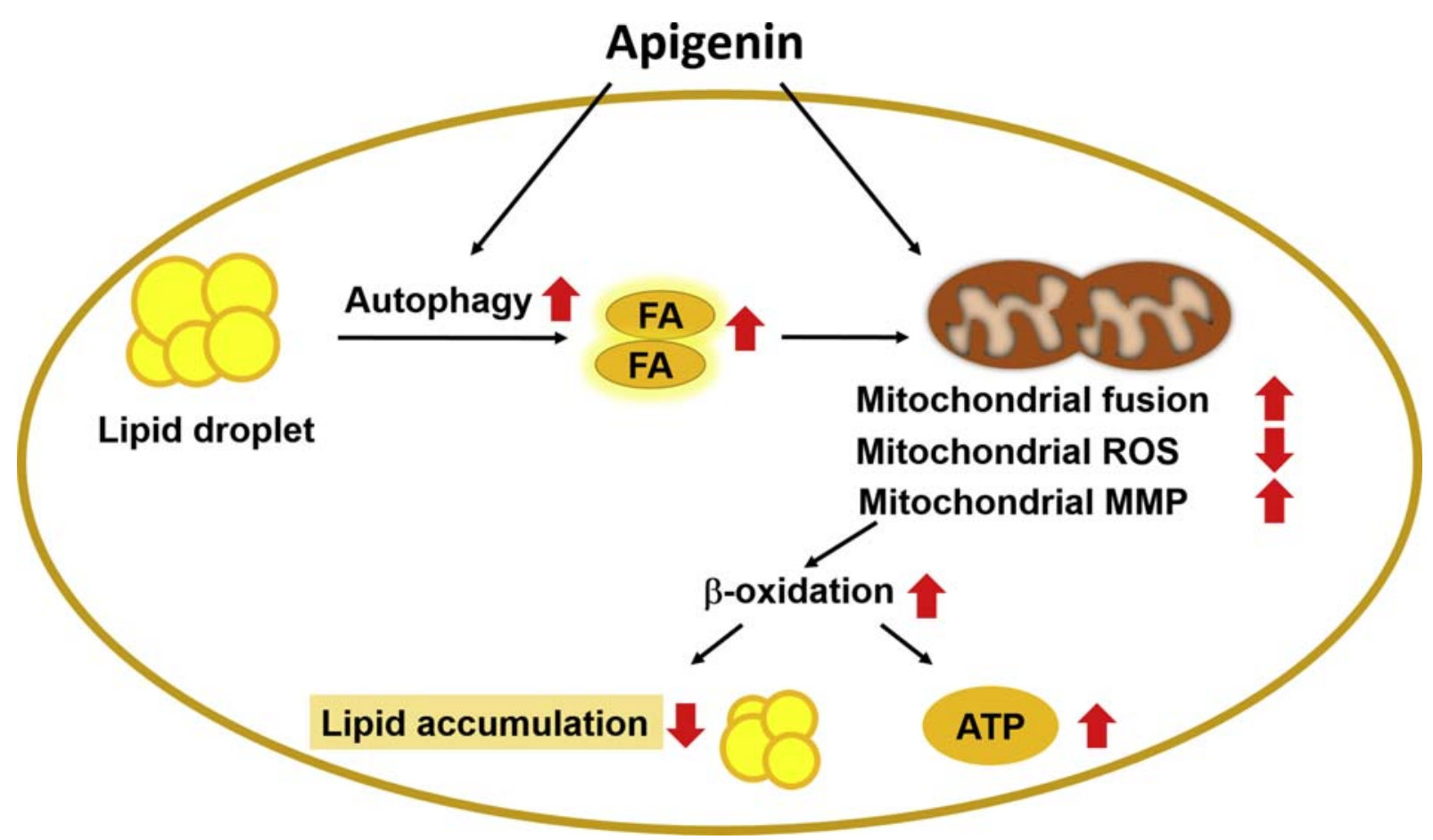

Fig. 11. Schematic illustration of the proposed molecular mechanisms by which apigenin protects the hepatocytes from the excess fatty acidinduced lipid accumulation. As shown, treatment with apigenin ameliorates OA-induced deregulation of lipid metabolism by activating the autophagy flux and rescuing mitochondrial dysfunction, leading to an increase in $\beta$-oxidation of fatty acids and ultimately decreasing the lipid accumulation in hepatocytes.

pathogenesis of metabolic diseases and to delineate the molecular mechanism underlying the apigeninconferred protection from lipid disorders. Using the obesity or NAFLD mouse models with genetic approaches will be helpful to clarify the effects and molecular mechanisms of apigenin on lipid disorders under pathological conditions. Moreover, we do not have clinical data to support our observations from in vitro studies. To this end, further in vivo studies or clinical trials describing the implications of apigenin in the deregulation of hepatic lipid metabolism and related metabolic diseases are warranted.

In conclusion, this study demonstrates the unique mechanisms of apigenin on hepatic lipid metabolism, which involves activation of autophagy flux, reduction of mitochondrial ROS production, rectification of mitochondrial function, and promotion of free fatty acid $\beta$-oxidation, and ultimately leads to alleviation of lipid accumulation in hepatocytes. Here, we provide new insight into the beneficial effects of apigenin on the deregulation of hepatic lipid metabolism, which broadens the biomedical implications of apigenin in the prevention or treatment of metabolic diseases.

\section{Conflicts of interest}

The authors declare no conflict of interest.

\section{Author contributions}

Man-Chen Hsu, Bei-Chia Guo, Chia-Hui Chen, and Po-An $\mathrm{Hu}$ performed the experiments and analyzed the data; TS Lee designed the experiments and wrote the paper.

\section{Acknowledgments}

This study was supported by grants from the Ministry of Science and Technology, Taiwan (106-2320-B-002-057-MY3, 106-2320-B-002-056, 1062811-B-002-146; 108-2811-B-002-542 and 108-2320-B$002-032-\mathrm{MY} 3)$. This article was subsidized for English editing by National Taiwan University under the Excellence Improvement Program for Doctoral Student (grant number 108-2926-I-002-002-MY4), sponsored by Ministry of Science and Technology, Taiwan. We thank the staff of the imaging core at the First Core Labs, National Taiwan University College of Medicine, for technical assistance. 


\section{Appendix A. Supplementary material}

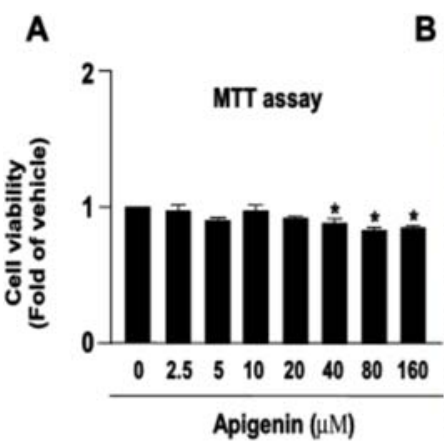

B
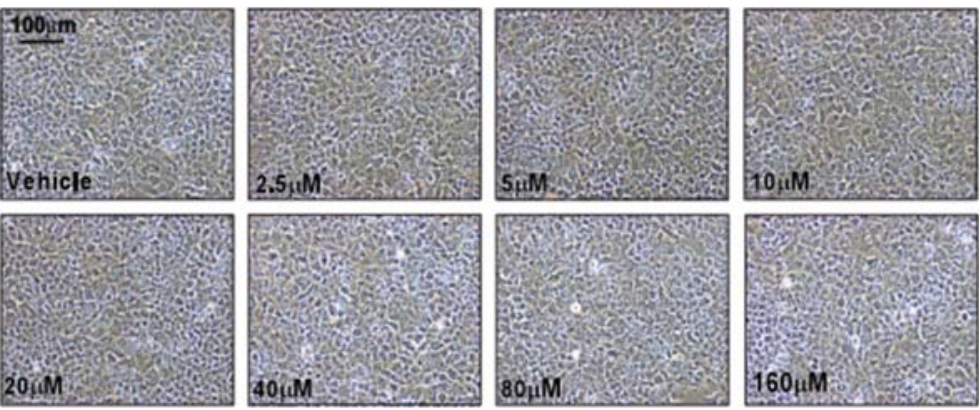

Fig. S1. Effect of increasing dose of apigenin on Huh7 cells. Huh7 cells were incubated with various concentrations $(0,2.5,5,10,20,40,80$ and 160 $\mu M)$ of apigenin for $18 \mathrm{~h}$. (A) Cell viability was determined by MTT assay. (B) Bright field images of cells treated with various dose of apigenin to observe the morphologic changes in Huh7 cells. Data are the mean \pm SEM from 5 independent experiments. $* P<0.05$ vs. the vehicle-treated group.

\section{A}

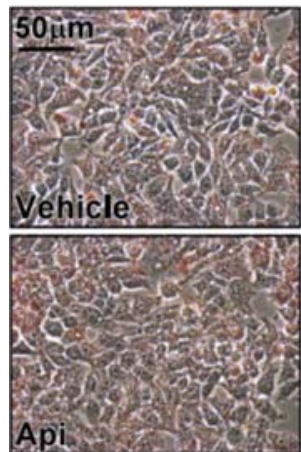

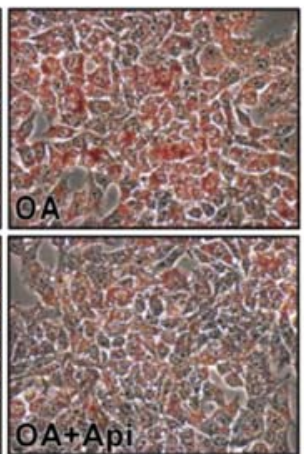

B
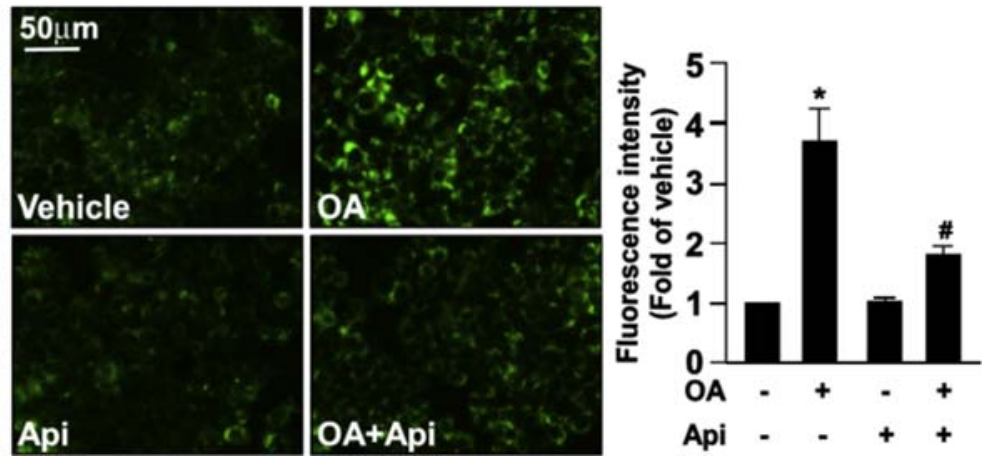

Fig. S2. Apigenin prevents oleic acid (OA)-induced lipid accumulation in HepG2 cells. HepG2 cells were pretreated with OA (125 $\mu g / m L)$ for 24 $h$ and then with or without of apigenin $(20 \mu M)$ for additional $18 \mathrm{~h}$. (A) Representative images of oil red $O$ staining of HepG2 cells. (B) Representative images and the quantitative intensity of Nile red staining. Data are the mean $\pm S E M$ from 5 independent experiments. ${ }^{*} P<0.05$ vs. the vehicletreated group; $\# P<0.05$ vs. the OA-treated group.

A

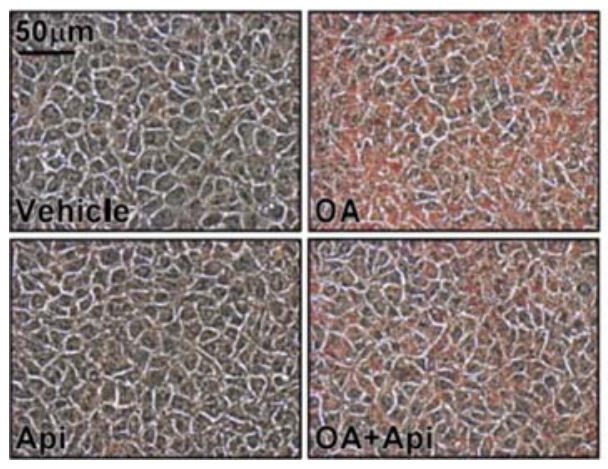

B

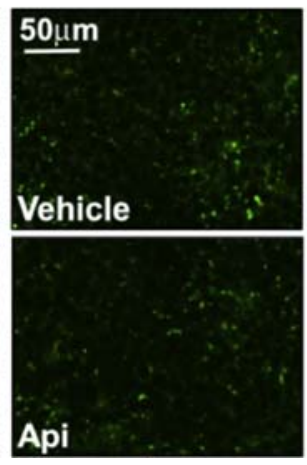

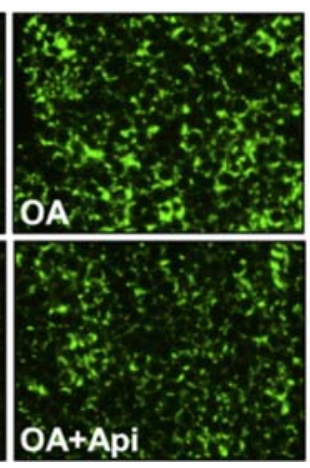

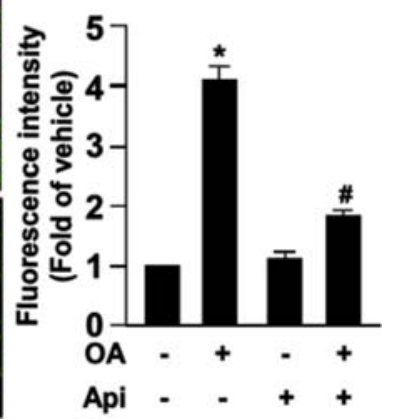

Fig. S3. Apigenin prevents oleic acid (OA)-induced lipid accumulation in AML 12 cells. AML 12 cells were pretreated with OA (125 $\mu \mathrm{g} / \mathrm{mL})$ for $24 \mathrm{~h}$ and then with or without of apigenin $(20 \mu \mathrm{M})$ for additional $18 \mathrm{~h}$. (A) Representative images of oil red O staining of AML 12 cells. (B) Representative images and the quantitative intensity of Nile red staining. Data are the mean \pm SEM from 5 independent experiments. $* P<0.05$ vs. the vehicle-treated group; ${ }^{\#}<0.05$ vs. the OA-treated group. 


\section{References}

[1] Tang D, Chen K, Huang L, Li J. Pharmacokinetic properties and drug interactions of apigenin, a natural flavone. Expert Opin Drug Metab Toxicol 2017;13:323-30.

[2] Miean KH, Mohamed S. Flavonoid (myricetin, quercetin, kaempferol, luteolin, and apigenin) content of edible tropical plants. J Agric Food Chem 2001;49:3106-12.

[3] Masuelli L, Benvenuto M, Mattera R, Di Stefano E, Zago E, Taffera G, et al. In Vitro and In Vivo anti-tumoral effects of the flavonoid apigenin in malignant mesothelioma. Front Pharmacol 2017;8:373.

[4] Ginwala R, Bhavsar R, Chigbu DI, Jain P, Khan ZK. Potential role of flavonoids in treating chronic inflammatory diseases with a special focus on the anti-inflammatory activity of apigenin. Antioxidants (Basel) 2019;8:35.

[5] Escande C, Nin V, Price NL, Capellini V, Gomes AP, Barbosa MT, et al. Flavonoid apigenin is an inhibitor of the $\mathrm{NAD}+$ ase CD38: implications for cellular NAD + metabolism, protein acetylation, and treatment of metabolic syndrome. Diabetes 2013;62:1084-93.

[6] Zhang L, Cheng X, Gao Y, Zheng J, Xu Q, Sun Y, et al. Apigenin induces autophagic cell death in human papillary thyroid carcinoma BCPAP cells. Food Funct 2015;6:3464-72.

[7] Charalabopoulos A, Davakis S, Lambropoulou M, Papalois A, Simopoulos C, Tsaroucha A. Apigenin exerts anti-inflammatory effects in an experimental model of acute pancreatitis by down-regulating TNF- $\alpha$. Vivo 2019;33:1133-41.

[8] Ren K, Jiang T, Zhou HF, Liang Y, Zhao GJ. Apigenin retards atherogenesis by promoting ABCA1-mediated cholesterol efflux and suppressing inflammation. Cell Physiol Biochem 2018;47:2170-84.

[9] Jung UJ, Cho YY, Choi MS. Apigenin ameliorates dyslipidemia, hepatic steatosis and insulin resistance by modulating metabolic and transcriptional profiles in the liver of high-fat diet-induced obese mice. Nutrients 2016;8:305.

[10] Feng X, Yu W, Li X, Zhou F, Zhang W, Shen Q, et al. Apigenin, a modulator of PPAR $\gamma$, attenuates HFD-induced NAFLD by regulating hepatocyte lipid metabolism and oxidative stress via Nrf2 activation. Biochem Pharmacol 2017; 136:136-49.

[11] Li Y, Ren L, Song G, Zhang P, Yang L, Chen X, et al. Silibinin ameliorates fructose-induced lipid accumulation and activates autophagy in HepG2 Cells. Endocr Metab Immune Disord Drug Targets 2019;19:632-642.

[12] Stacchiotti A, Grossi I, García-Gómez R, Patel GA, Salvi A, Lavazza A, et al. Melatonin effects on non-alcoholic fatty liver disease are related to microRNA-34a-5p/Sirt1 axis and autophagy. Cells 2019;8:1053.

[13] Tanida I, Ueno T, Kominami E. LC3 and autophagy. Methods Mol Biol 2008;445:77-88.

[14] Singh R, Cuervo AM. Lipophagy: connecting autophagy and lipid metabolism. Int J Cell Biol 2012;2012:282041.

[15] Wei Y, Rector RS, Thyfault JP, Ibdah JA. Nonalcoholic fatty liver disease and mitochondrial dysfunction. World J Gastroenterol 2008;14:193-9.

[16] Pagliarini DJ, Rutter J. Hallmarks of a new era in mitochondrial biochemistry. Genes Dev 2013;27:2615-27.

[17] Leipnitz G, Mohsen AW, Karunanidhi A, Seminotti B, Roginskaya VY, Markantone DM, et al. Evaluation of mitochondrial bioenergetics, dynamics, endoplasmic reticulum-mitochondria crosstalk, and reactive oxygen species in fibroblasts from patients with complex I deficiency. Sci Rep 2018;8:1165.

[18] Youle RJ, van der Bliek AM. Mitochondrial fission, fusion, and stress. Science 2012;337:1062-5.

[19] Dai W, Jiang L. Dysregulated mitochondrial dynamics and metabolism in obesity, diabetes, and cancer. Front Endocrinol (Lausanne) 2019;10:570.

[20] Begriche K, Massart J, Robin MA, Bonnet F, Fromenty B. Mitochondrial adaptations and dysfunctions in nonalcoholic fatty liver disease. Hepatology 2013;58:1497-507.
[21] Mansouri A, Gattolliat CH, Asselah T. Mitochondrial dysfunction and signaling in chronic liver diseases. Gastroenterology 2018;155:629-47.

[22] Itami N, Shirasuna K, Kuwayama T, Iwata H. Palmitic acid induces ceramide accumulation, mitochondrial protein hyperacetylation, and mitochondrial dysfunction in porcine oocytes. Biol Reprod 2018;98:644-53.

[23] Mishra P, Chan DC. Metabolic regulation of mitochondrial dynamics. J Cell Biol 2016;212:379-87.

[24] Zeng P, Liu B, Wang Q, Fan Q, Diao JX, Tang J, et al. Apigenin attenuates atherogenesis through inducing macrophage apoptosis via inhibition of AKT Ser473 phosphorylation and downregulation of plasminogen activator inhibitor-2. Oxid Med Cell Longev 2015;2015:379538.

[25] Lim H, Lim YM, Kim KH, Jeon YE, Park K, Kim J, et al. A novel autophagy enhancer as a therapeutic agent against metabolic syndrome and diabetes. Nat Commun 2018;9:1438.

[26] Item F, Wueest S, Lemos V, Stein S, Lucchini FC, Denzler R, et al. Fas cell surface death receptor controls hepatic lipid metabolism by regulating mitochondrial function. Nat Commun 2017;8:480.

[27] Chen X, Xu H, Yu X, Wang X, Zhu X, Xu X. Apigenin inhibits in vitro and in vivo tumorigenesis in cisplatin-resistant colon cancer cells by inducing autophagy, programmed cell death and targeting $\mathrm{m}$-TOR/PI3K/Akt signalling pathway. J BUON 2019;24:488-493.

[28] Bujak AL, Crane JD, Lally JS, Ford RJ, Kang SJ, Rebalka IA, et al. AMPK activation of muscle autophagy prevents fastinginduced hypoglycemia and myopathy during aging. Cell Metab 2015;21:883-90.

[29] Viollet B, Guigas B, Leclerc J, Hébrard S, Lantier L, Mounier R, et al. AMP-activated protein kinase in the regulation of hepatic energy metabolism: from physiology to therapeutic perspectives. Acta Physiol (Oxf) 2009;196:81-98.

[30] Hahm JR, Noh HS, Ha JH, Roh GS, Kim DR. Alpha-lipoic acid attenuates adipocyte differentiation and lipid accumulation in 3T3-L1 cells via AMPK-dependent autophagy. Life Sci 2014;100:125-32.

[31] Huang Q, Wang T, Yang L, Wang HY. Ginsenoside Rb2 alleviates hepatic lipid accumulation by restoring autophagy via induction of Sirt1 and activation of AMPK. Int J Mol Sci 2017;18:1063.

[32] Lu J, Meng Z, Cheng B, Liu M, Tao S, Guan S. Apigenin reduces the excessive accumulation of lipids induced by palmitic acid via the AMPK signaling pathway in HepG2 cells. Exp Ther Med 2019;18:2965-71.

[33] Chen J, Chen J, Huang J, Li Z, Gong Y, Zou B, Liu X, Ding L, Li P, Zhu Z, Zhang B, Guo H, Cai C, Li J. HIF-2 $\alpha$ upregulation mediated by hypoxia promotes NAFLD-HCC progression by activating lipid synthesis via the PI3K-AKT-mTOR pathway. Aging (Albany NY) 2019;11:10839-60.

[34] Zhou W, Ye S. Rapamycin improves insulin resistance and hepatic steatosis in type 2 diabetes rats through activation of autophagy. Cell Biol Int 2018;42:1282-91.

[35] Farah BL, Landau DJ, Sinha RA, Brooks ED, Wu Y, Fung SYS, et al. Induction of autophagy improves hepatic lipid metabolism in glucose-6-phosphatase deficiency. J Hepatol 2016;64:370-9.

[36] Upadhyay KK, Jadeja RN, Vyas HS, Pandya B, Joshi A Vohra A, et al. Carbon monoxide releasing molecule-A1 improves nonalcoholic steatohepatitis via Nrf2 activation mediated improvement in oxidative stress and mitochondrial function. Redox Biol 2020;28:101314.

[37] Jornayvaz FR, Shulman GI. Regulation of mitochondrial biogenesis. Essays Biochem 2010;47:69-84.

[38] Lee H, Yoon Y. Mitochondrial fission and fusion. Biochem Soc Trans 2016;44:1725-35.

[39] Zorov DB, Juhaszova M, Sollott SJ. Mitochondrial reactive oxygen species (ROS) and ROS-induced ROS release. Physiol Rev 2014;94:909-50.

[40] Teodoro JS, Palmeira CM, Rolo AP. Mitochondrial membrane potential $(\Delta \Psi)$ fluctuations associated with the metabolic states of mitochondria. Methods Mol Biol 2018;1782: 109-19. 
[41] Madamanchi NR, Runge MS. Mitochondrial dysfunction in atherosclerosis. Circ Res 2007;100:460-73.

[42] Mi Y, Qi G, Gao Y, Li R, Wang Y, Li X, et al. (-)-Epigallocatechin-3-gallate ameliorates insulin resistance and mitochondrial dysfunction in HepG2 Cells: Involvement of Bmal1. Mol Nutr Food Res 2017;61. https://doi.org/10.1002/ mnfr.201700440.

[43] Yang CS, Wang H, Sheridan ZP. Studies on prevention of obesity, metabolic syndrome, diabetes, cardiovascular diseases and cancer by tea. J Food Drug Anal 2018;26:1-13. https://doi.org/10.1016/j.jfda.2017.10.010.

[44] Choi WH, Son HJ, Jang YJ, Ahn J, Jung CH, Ha TY. Apigenin ameliorates the obesity-induced skeletal muscle atrophy by attenuating mitochondrial dysfunction in the muscle of obese mice. Mol Nutr Food Res 2017;61. https://doi.org/ 10.1002/mnfr.201700218.

[45] Rehman K, Haider K, Jabeen K, Akash MSH. Current perspectives of oleic acid: Regulation of molecular pathways in mitochondrial and endothelial functioning against insulin resistance and diabetes. Rev Endocr Metab Disord 2020. https://doi.org/10.1007/s11154-020-09549-6.

[46] Morrow NM, Burke AC, Samsoondar JP, Seigel KE, Wang A, Telford DE, et al. The citrus flavonoid nobiletin confers protection from metabolic dysregulation in high-fat-fed mice independent of AMPK. J Lipid Res 2020;61:387-402.

[47] Fu PP, Xia Q, Hwang HM, Ray PC, Yu H. Mechanisms of nanotoxicity: generation of reactive oxygen species. J Food Drug Anal 2014;22:64-75. https://doi.org/10.1016/j.jfda.2014.01.005.

[48] Mahjoub S, Masrour-Roudsari J. Role of oxidative stress in pathogenesis of metabolic syndrome. Caspian J Intern Med 2012;3:386-96.

[49] Boren J, Brindle KM. Apoptosis-induced mitochondrial dysfunction causes cytoplasmic lipid droplet formation. Cell Death Differ 2012;19:1561-70.

[50] Forrester SJ, Kikuchi DS, Hernandes MS, Xu Q, Griendling KK. Reactive oxygen species in metabolic and inflammatory signaling. Circ Res 2018;122:877-902.

[51] Bhatti JS, Bhatti GK, Reddy PH. Mitochondrial dysfunction and oxidative stress in metabolic disorders - A step towards mitochondria based therapeutic strategies. Biochim Biophys Acta Mol Basis Dis 2017;1863:1066-77.

[52] Zhong Y, Jin C, Gan J, Wang X, Shi Z, Xia X, et al. Apigenin attenuates patulin-induced apoptosis in HEK293 cells by modulating ROS-mediated mitochondrial dysfunction and caspase signal pathway. Toxicon 2017;137:106-13. 